\title{
Deregulated expression of superoxide dismutase-2 correlates with different stages of cervical neoplasia
}

\author{
Lara Termini $^{\mathrm{a}, \mathrm{d}, *}$, Adhemar Longatto Filho $^{\mathrm{b}, \mathrm{e}}$, Paulo Cesar Maciag $^{\mathrm{d}}$, Daniela Etlinger ${ }^{\mathrm{c}}$, \\ Venâncio Avancini Ferreira Alves ${ }^{\mathrm{e}}$, Suely Nonogaki ${ }^{\mathrm{c}}$, Fernando Augusto Soares ${ }^{\mathrm{f}, \mathrm{g}}$ and \\ Luisa Lina Villa ${ }^{a, d}$ \\ ${ }^{\mathrm{a}}$ Ludwig Institute for Cancer Research - Hospital Alemão Oswaldo Cruz, São Paulo (SP), Brazil \\ ${ }^{\mathrm{b}}$ Life and Health Sciences Research Institute, School of Health Sciences, University of Minho, Campus de Gualtar, \\ Braga, Portugal \\ ${ }^{\mathrm{c}}$ Pathology Division, Adolfo Lutz Institute, São Paulo (SP), Brazil \\ ${ }^{\mathrm{d}}$ HPV Institute - INCT-HPV, Santa Casa de Misericórdia, São Paulo (SP), Brazil \\ ${ }^{\mathrm{e}}$ Laboratory of Medical Investigation (LIM) 14, Department of Pathology, University of São Paulo, School of \\ Medicine, São Paulo(SP), Brazil \\ ${ }^{\mathrm{f}}$ Department of Anatomic Pathology, Hospital do A. C. Camargo, São Paulo (SP), Brazil \\ ${ }^{\mathrm{g}}$ Department of General Pathology, Faculty of Dentistry of the University of São Paulo, São Paulo, Brazil
}

\begin{abstract}
Objective: Superoxide dismutase-2 (SOD2) is considered one of the most important antioxidant enzymes that regulate cellular redox state in normal and tumorigenic cells. Overexpression of this enzyme may be involved in carcinogenesis, particularly in lung, gastric, colorectal and breast cancer.

Methods: In the present study, we have evaluated SOD2 protein levels by immunohistochemistry (IHC) in 331 cervical histological samples including 31 low-grade cervical intraepithelial neoplasia (LSIL), 51 high-grade cervical intraepithelial neoplasia (HSIL), 197 squamous cervical carcinomas (SCC) and 52 cervical adenocarcinomas (ADENO).

Results: We observed that SOD2 staining increases with cervical disease severity. Intense SOD2 staining was found in $13 \%$ of LSIL, $25.5 \%$ of HSIL and $40 \%$ of SCC. Moreover, $65.4 \%$ of ADENO exhibited intense SOD2 staining.

Conclusions: Differences in the expression of SOD2 could potentially be used as a biomarker for the characterization of different stages of cervical disease.
\end{abstract}

Keywords: Human papillomavirus, Tissue Microarray, Biomarker, Cervical Neoplasia, Superoxide Dismutase-2

\section{Introduction}

The natural history of cervical cancer is associated with persistent infection of human papillomavirus (HPV), which is now recognized as a necessary cause for this disease. The identification of pre-invasive le-

*Corresponding author: Lara Termini, PhD, Ludwig Institute for Cancer Research, Laboratory of Virology, Hospital Alemão Oswaldo Cruz, Rua João Julião, $2451^{\circ}$ andar, 01323-903 - São Paulo - SP, Brazil. Tel.: +55 1135490461 ; E-mail: 1termini@vrg.ludwig.org.br. sions and associated markers of progression has an important value for screening purposes and prognostic evaluation of these lesions [7]. Cervical carcinoma and its precursor lesions are important problems of public health, carrying high annual morbidity and mortality in developing countries. Most of the available molecular tests designed to identify high risk HPV infection can significantly improve the cervical cancer screening $[8$, 28]. There are 2 main histological types of cervical cancer: squamous cell carcinomas (SCC) and adenocarcinomas (ADENO). Among the cervical lesions induced by HPV, ADENO are one of the most challeng- 
ing types to be understood and recognized in cytological daily routine examination. This difficulty is due to the scarcity of cases and some particular characteristics related to the neoplastic cells differentiation such as absence of prominent nucleoli, lack of acinar arrangement and other confounding morphological parameters that resemble high-grade squamous intraepithelial lesion (HSIL). Additionally, ADENO are difficult to be diagnosed because several benign lesions can mimic malignant and premalignant endocervical glandular lesions [24,26]. Furthermore, certain variants of endocervical ADENO have unique biological behavior and should be considered as an individual entity, differing in terms of prognosis and therapy [38].

In spite of substantial progress and opportunities for primary and secondary cervical cancer prevention, important queries still remain to be clarified regarding the molecular mechanisms controlling cervical cancer progression [30]. Thus, most of the current efforts in this field have been dedicated to the investigation of genes that are differentially expressed in cervical cancer when compared to their normal tissue counterparts. However, robust findings have shown that no more than a subset of these genes is associated with cancer progression and metastatic phenotype [10]. Using microarray approach we have compared the global transcription profile of normal and HPV-immortalized keratinocytes, upon TNF $\alpha$ treatment. Among the differentially expressed genes associated with inflammatory response we have identified superoxide dismutase-2 (SOD2) transcript [34].

Superoxide dismutases (SOD) are metalloenzymes that act in the cellular antioxidant system and are ubiquitously expressed in prokaryotic and eukaryotic cells. These proteins protect redox sensitive cellular machinery from damage by catalyzing the disproportionation of superoxide anion to oxygen and hydrogen peroxide [18]. Three distinct isoforms of SOD have been identified and characterized in mammals: copper-zinc superoxide dismutase (encoded by the SODl gene), manganese superoxide dismutase (encoded by the SOD2 gene) and extracellular superoxide dismutase (encoded by the SOD 3 gene). Members from the SOD family have similar functions, but features like their gene chromosome localization, protein structure, metal cofactor condition and cellular compartmentalization may differ from one to another [6, 25]. SOD2 is found exclusively in the mitochondrial matrix and is an evolutionary conserved enzyme in a variety of organisms [12].

SOD alterations are associated with a number of diseases including arteriosclerosis, diabetes mellitus and
Down syndrome [6]. Moreover, several studies have shown that SOD2 protein expression is up-regulated in cancer cells when compared to their normal counterparts, including colorectal [36], lung [11] and gastric/esophageal cancers $[12,15,17,23]$. However, the relationship between SOD2 expression and cervical carcinogenesis has not been addressed.

In the present study we have analyzed SOD2 expression in 331 cervical tissue samples including lowgrade squamous intraepithelial lesion (LSIL), highgrade squamous intraepithelial lesion (HSIL) and invasive carcinomas. We observed that SOD2 levels increase in SCC and in ADENO samples as compared to LSIL.

\section{Materials and methods}

\subsection{Tissue samples and tissue microarray (TMA) construction}

Cervical tissue samples were obtained from the Department of Anatomic Pathology, Medical and Research Centre, Hospital A. C. Camargo, from the Pathology Division, Adolfo Lutz Institute, and from the Laboratory of Medical Investigation (LIM) 14, Department of Pathology, University of São Paulo, School of Medicine, from São Paulo, Brazil. Ethical approval for this study was granted by local research ethics committees. In this study, 331 cervical samples corresponding to $31 \mathrm{LSIL}, 51 \mathrm{HSIL}, 197 \mathrm{SCC}$ and 52 cervical ADENO were used to construct 3 TMA (Tissue Microarray) platforms. Low-grade squamous intraepithelial lesions (LSIL) and high-grade squamous intraepithelial lesion (HSIL) samples were spotted in duplicate.

SCC and ADENO samples belong to a retrospective cohort study in which patients diagnosed with carcinoma of the uterine cervix underwent radical surgical treatment at Hospital A. C. Camargo between 1980 and 1999. The inclusion criteria were: 1) Histological proof of invasive carcinoma of the uterine cervix; 2) Clinical staging IB and IIA, according to the criteria established by FIGO; 3) Absence of any previous treatment; 4) Surgical treatment performed by means of Piver-Rutledge class II or III radical hysterectomy and pelvic lymphnodectomy.

Fixed and paraffin embedded tissues were first sectioned in $3 \mu \mathrm{m}$ sections, which were hematoxylin-eosin stained for selection of the appropriated tissue area. The correspondent selected areas of each tissue sample were then collected from the donor embedded tissues 


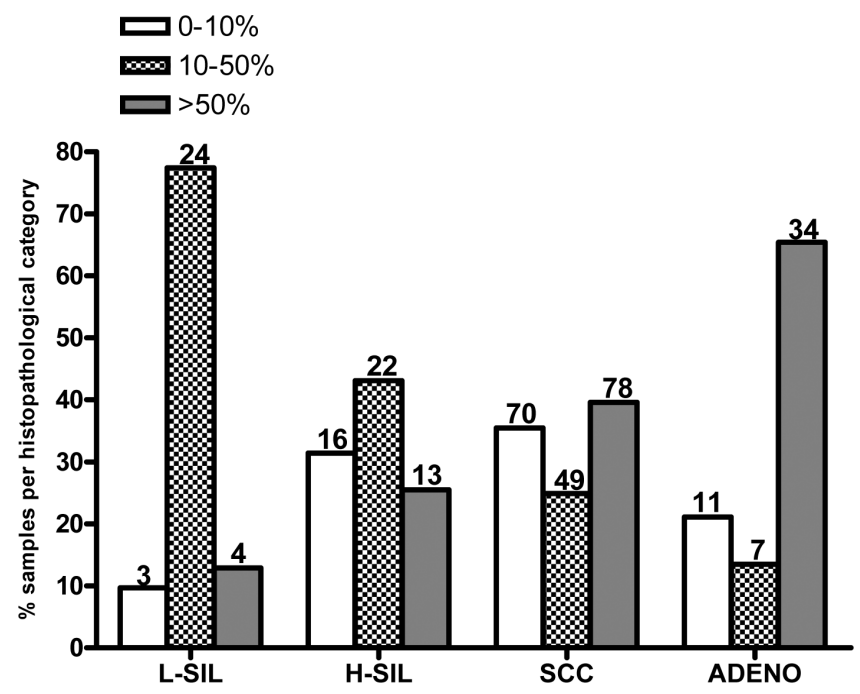

Fig. 1. Distribution of SOD2 staining according to diagnostic classification. Samples were grouped depending on the staining intensity as negative or weak $(<10 \%$ positivity), moderate $(10-50 \%$ positivity) and intense $(>50 \%$ positivity). L-SIL: low-grade squamous intraepithelial lesions; H-SIL: high-grade squamous intraepithelial lesions; SCC: squamous cervical carcinomas; ADENO: cervical adenocarcinomas. Numbers on top of each bar correspond to the number of samples in each category.

with a $1 \mathrm{~mm}$ gauge cylinder and transferred to a receptor block using a Manual Tissue Microarrayer (Beecher Instruments, Sun Prairie, WI, USA). TMA $5 \mu \mathrm{m} \mathrm{sec-}$ tions were cut from the receptor block and processed as described below.

\subsection{Immunohistochemical SOD2 detection}

After deparaffinization in xylene and rehydration in alcohol, antigen retrieval was performed by incubation in boiled citrate buffer $\mathrm{pH} 6.0$ in a pressure cooker for 4 minutes. Endogenous peroxidase activity was inactivated with $3 \%$ hydrogen peroxide. Nonspecific avidin-binding was also blocked (DAKO, X0590, Carpinteria, CA, USA). Samples were incubated with an anti-SOD2 polyclonal antibody (Santa Cruz Biotechnology, sc-18504, Santa Cruz, CA, USA), 1:100 in $1 \%$ bovine serum albumin-phosphate buffered solution for 30 minutes at $37^{\circ} \mathrm{C}$ and for 18 hours at $4^{\circ} \mathrm{C}$. The slides were then incubated for 30 minutes at $37^{\circ} \mathrm{C}$ with secondary biotinylated rabbit anti-goat IgG, (Vector, BA5000, Burlingame, CA, USA) diluted 1:500, followed by incubation for 30 minutes at $37^{\circ} \mathrm{C}$ with the complex streptavidin and biotinylated peroxidase (StreptABComplex/HRP Duet Mouse/Rabbit, DakoCytomation, K0492, Glostrup, Denmark), diluted 1:200. Slides were developed using $100 \mathrm{mg}$ of 3,3'diaminobenzidine tetrahydrochloride (Sigma, D-5637, St Louis, MO, USA), $6 \% \mathrm{H}_{2} \mathrm{O}_{2}$, and dimethyl sulphoxide and counterstained with Harris' hematoxylin. Sec- tions derived from an ovarian papillary serous adenocarcinoma were used as a positive control and were incubated in the absence or in the presence of anti-SOD2 antibody. Sections of normal cervical tissues were used as negative control.

\subsection{Evaluation of tissue staining}

The positive immunohistochemical reaction was evaluated considering the percentage of epithelial stained cells in each representative histopathological category (LSIL, HSIL, SCC and ADENO). The immunohistochemical reactions were performed in duplicate on sections obtained from the TMA blocks. Only tissue cores with more than $25 \%$ of cervical epithelium were analyzed. The evaluation was performed by examining the epithelium of the whole punch from each sample present in the TMA platforms under X200 magnification. As previously reported [35], positive reactions were semi quantitatively scored as "negative/weak" ( $<10 \%$ stained cells), "moderate' (10$50 \%$ stained cells) and "intense" (>50\% stained cells) (Fig. 1). Immunohistochemical evaluation was performed independently and blindly by two observers (ALF and FAS); the very few discordant results were discussed by both observers and a final score was established.

\subsection{Statistical analysis}

Comparisons among proportions were done using the Fisher exact test and the linear-by-linear association 
Table 1

Association of intense SOD2 positivity and grade of cervical lesion

\begin{tabular}{lccc}
\hline \multicolumn{4}{c}{ SOD2 cellular staining } \\
\hline Diagnostic Classification & $<50 \%$ & $>50 \%$ & OR $(95 \% \mathrm{CI})^{*}$ \\
\hline LSIL & $87 \%(27)$ & $13 \%(4)$ & 1,0 (ref.) \\
HSIL & $74.5 \%(38)$ & $25.5 \%(13)$ & $2.3(0.6-7.8)$ \\
SCC & $60 \%(119)$ & $40 \%(78)$ & $4.4(1.4-13.1)^{*}$ \\
ADENO & $34.6 \%(18)$ & $65.4 \%(34)$ & $12.7(3.8-42.1)^{* *}$ \\
\hline$*$ p-
\end{tabular}

${ }^{*}$ p-value $<0,004 ;{ }^{* *}$ p-value $<0.001$ (Fisher's exact test). Numbers between parentheses correspond to samples contained in each category.

test was used to evaluate trends among proportions. Samples diagnosed as LSIL were used as the reference category for comparisons with HSIL, SCC and ADENO. For the statistical analysis, reactions were scored as less than $50 \%$ stained cells (negative to moderate expression) and more than $50 \%$ stained cells (intense expression). The 'negative or weak' $(<10 \%$ stained cells) samples in the LSIL group were very limited $(n=3)$, and this category was combined to the moderate (10-50\% stained cells) group to provide more robust estimates. Odds Ratios (OR) and 95\% confidence intervals were estimated using $2 \times 2$ contingency tables. All statistical analyses were done with the Statistical Package for the Social Sciences 15.0 software. Statistical significance was based on a value of $p<$ 0.05 .

\section{Results}

We have analyzed SOD2 expression in 331 cervical samples comprising 31 (9.3\%) LSIL, 51 (15.4\%) HSIL, 197 (59.5\%) SCC and 52 (15.8\%) ADENO. Overall, the TMA platforms used in this study were representative of different cervical lesions. An evaluation with hematoxylin-eosin staining revealed adequate results for the majority of specimens and only cores with more than $25 \%$ of cervical epithelium tissue were analyzed. Unfortunately, such tissues were not amenable for amplification of HPV genomes (or housekeeping genes, such as globin). Therefore, no data on HPV status is available in this study.

We observed a clear pattern where the percentage of samples exhibiting intense staining for SOD2 (> $50 \%$ positive cells) was increased in HSIL (25.5\%, $p=0.262)$ and SCC $(40 \%, p<0.004)$, as compared to LSIL (13\%) (Fig. 2 and Table 1). Furthermore, when we analyzed ADENO we observed that $65.4 \%$ of the specimens exhibited more than $50 \%$ of positive cell staining for SOD2 $(p<0.001)$ (Fig. 2). Finally, we observed a significant positive association between high SOD2 expression level ( $>50 \%$ stained cells) and the grade of cervical lesion. Intense positivity for SOD2 ( $>50 \%$ stained cells) was significantly associated with SCC $(\mathrm{OR}=4.4, \mathrm{CI}: 1.4-13.1)$, when compared to LSIL group. Importantly, the association with SOD2 positivity was particularly stronger with ADENO (OR $=12.7$, CI: 3.8-42.1). No significant difference in SOD2 expression was observed between the LSIL and HSIL samples in this study (OR $=2.3, \mathrm{CI}$ : 0.6-7.8) (Table 1).

Figure 3 shows a representative immunostaining for SOD2 in different cervical lesions and tumor samples exhibiting $<50 \%$ (Fig. 3a, c, e, and g) or $>50 \%$ (Fig. 3b, d, f, and h) stained cells. SOD2 positive cells display preferentially a granular cytoplasmic staining pattern. Intensive staining in HSIL, SCC and ADENO displayed a strong cytoplasmic SOD2 staining throughout the lesion while no staining was observed in the stromal cells. However, in some cases SOD2 expression was also observed in inflammatory cells infiltrating both epithelium and stroma (Fig. 3b). In general, normal squamous and glandular epithelial cells are negative for SOD2 staining. In some areas of normal squamous epithelium, however, we observed focal staining in the basal and/or suprabasal cell layers (data not shown).

\section{Discussion}

The role of SOD2 in cancer onset and progression is characterized by a complex net of events that underscores the multifactorial nature of this enzyme and its effects on important cellular processes such as cell proliferation, growth and differentiation. In vitro studies have demonstrated that overexpression of members of the SOD family correlates with increased cell differentiation, decreased cell growth and proliferation and, remarkably, reversion of malignant phenotype [19].The exact functions of SOD2 and redox state in neoplasias are not completely understood. Theoretically, reduc- 


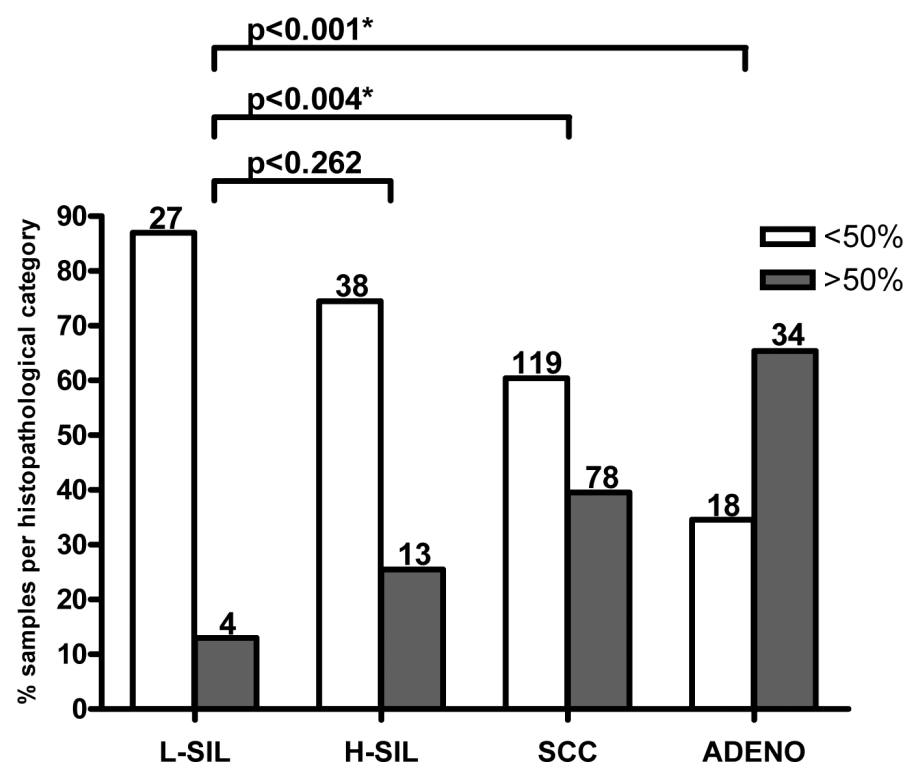

Fig. 2. Distribution of SOD2 staining according to diagnostic classification. Samples were grouped depending on the staining intensity as negative, weak or moderate $(<50 \%$ positivity) and intense ( $>50 \%$ positivity). LSIL: low-grade squamous intraepithelial lesions; HSIL: high-grade squamous intraepithelial lesions; SCC: squamous cervical carcinomas; ADENO: cervical adenocarcinomas. Numbers on top of each bar correspond to the number of samples in each category. ${ }^{*}$ p-values.

ing the oxidative stress by increasing SOD2 levels may prevent DNA injury and consequently cancer development. In fact, a protective role of SOD2 against tumor progression in several transformed cell lines has been reported $[4,5,21,27,37,39]$. On the other hand, reduction of oxidative stress in tumor cells may preclude the accumulation of reactive oxygen species (ROS), preventing programmed cell death (PCD) or necrosis onset, favoring tumor progression $[12,25]$. This view is supported by in vivo analyses that correlate SOD2 overexpression with worse prognosis in solid tumors. Several reports have shown increased SOD2 levels in many malignancies, including lung [20,33], colorectal [16,36], gastric and esophageal [13,17], brain [9], oral [22] and skin carcinomas [32], often associated with metastasis and poor prognosis. In contrast, SOD2 reduction was also observed in prostatic carcinomas when compared to normal tissues [1,3].

These apparent paradoxes have been attributed, in part, to the variation of malignant cells oxidative system according to each specific microenvironment [19]. Recently, it was demonstrated that intracellular oxidizing state is enhanced in breast cancer patients while the antioxidant defense mechanism is weakened. This enhanced activity is associated with the increasing severity of cancer as determined by TNM staging. However, the study of SOD2 levels in breast cancer generated conflicting results, with some data showing in- creased SOD2 [2], whilst others clearly demonstrated a decrease in SOD2 levels [31].

In this study, we examined the expression of SOD2 in a large set of cervical samples, including LSIL, HSIL, SCC and ADENO by IHC. We observed that HSIL and invasive cancer exhibited higher SOD2 levels than LSIL samples. For comparisons, the latter were chosen as reference category because the majority of LSIL has a benign evolution, represent HPV productive infections and regress spontaneously $[14,29]$. The trend observed between SOD2 positivity and disease severity was statistically significant $(p<0.004)$.

In most LSIL, we observed no or low SOD2 expression (Fig. 3a). On the other hand, HSIL, SCC and ADENO included in the $>50 \%$ staining category exhibited predominantly cytoplasmic granulated-like deposits in all the layers of the epithelium (Fig. 3d, $\mathrm{f}$ and h). Ongoing studies will determine whether the different patterns of SOD2 expression could be associated with HPV type or viral genome integration.

This is the first study analyzing the expression of SOD2 in a large series of cervical samples. Our results show a trend between SOD2 positivity and severity of cervical disease including squamous and glandular invasive lesions. We can speculate that increased SOD2 levels in tumorigenic cells may confer an adaptive advantage favoring tumor establishment and progression. We are currently analyzing the correlation be- 


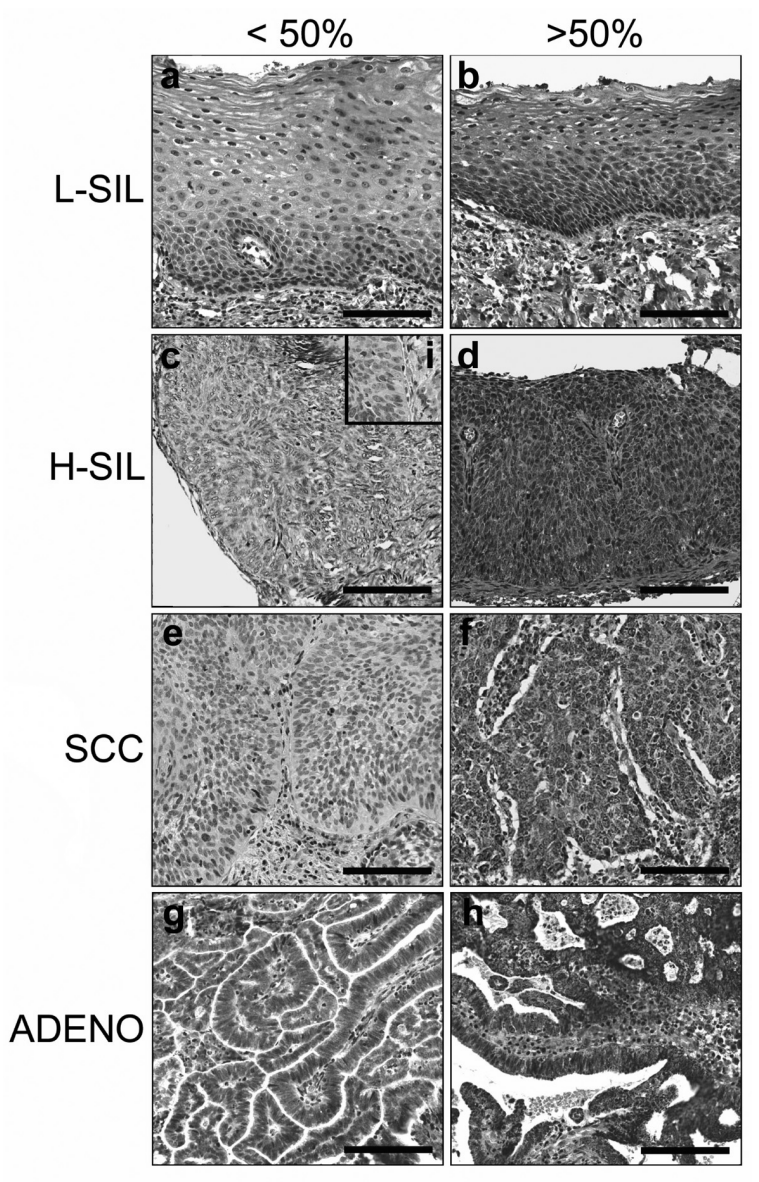

Fig. 3. Immunohistochemical analysis of SOD2 expression in cervical samples. Representative immunoreactivity of SOD2 in low-grade squamous intraepithelial lesions (LSIL) ( $a$ and b); high-grade squamous intraepithelial lesions (HSIL) (c and d); squamous cervical carcinoma (SCC) (e and f) and adenocarcinomas (ADENO) ( $g$ and $\mathrm{h}$ ). Less than $50 \%$ of stained cells were observed in (a), (c), (e) and (g) while (b), (d), (f) and (h) showed more than $50 \%$ of stained cells. Samples incubated in the absence of primary antibody exhibited no immunostaining [inset in (i)]. Bar $=$ $100 \mu \mathrm{m}$. (Colours are visible in the online version of the article; http://dx.doi.org/10.3233/DMA-2011-0784)

tween SOD2 expression, HPV DNA presence and clinical prognosis in a larger number of HPV-typed LSIL, HSIL, SCC and ADENOS samples. These data may contribute to determine the role of SOD2 in cervical cancer development, identification and progression.

\section{Acknowledgements}

We are grateful to Carlos Ferreira do Nascimento and Severino Ferreira from the Hospital A.C. Camargo for technical assistance. We would like to thank Dr.
Enrique Boccardo, Dr. Ismar Haga and Dr. Ana Paula Lepique for critical review of the manuscript and Dr. Erico Tosoni Costa for assistance in figure preparation. Dr. Lara Termini was a Post-Doctoral fellow from Fundação de Amparo à Pesquisa do Estado de São Paulo (grant 05/57274-9).

\section{References}

[1] A.M. Baker, L.W. Oberley and M.B. Cohen, Expression of antioxidant enzymes in human prostatic adenocarcinoma, Prostate 32 (1997), 229-233.

[2] M.S. Bianchi, N.O. Bianchi and A.D. Bolzan, Superoxide dismutase activity and superoxide dismutase-1 gene methylation in normal and tumoral human breast tissues, Cancer Genet Cytogenet 59 (1992), 26-29.

[3] D.G. Bostwick, E.E. Alexander, R. Singh, A. Shan, J. Qian, R.M. Santella, L.W. Oberley, T. Yan, W. Zhong, X. Jiang and T.D. Oberley, Antioxidant enzyme expression and reactive oxygen species damage in prostatic intraepithelial neoplasia and cancer, Cancer 89 (2000), 123-134.

[4] S.L. Church, J.W. Grant, L.A. Ridnour, L.W. Oberley, P.E. Swanson, P.S. Meltzer and J.M. Trent, Increased manganese superoxide dismutase expression suppresses the malignant phenotype of human melanoma cells, Proc Natl Acad Sci USA 90 (1993), 3113-3117.

[5] J.J. Cullen, C. Weydert, M.M. Hinkhouse, J. Ritchie, F.E. Domann, D. Spitz and L.W. Oberley, The role of manganese superoxide dismutase in the growth of pancreatic adenocarcinoma, Cancer Res 63 (2003), 1297-1303.

[6] V.C. Culotta, M. Yang and T.V. O'Halloran, Activation of superoxide dismutases: putting the metal to the pedal, Biochim Biophys Acta 1763 (2006), 747-758.

[7] E.L. Franco and J. Cuzick, Cervical cancer screening following prophylactic human papillomavirus vaccination, Vaccine 26 (2008), 16-23.

[8] J.D. Goldhaber-Fiebert, N.K. Stout, J.A. Salomon, K.M. Kuntz and S.J. Goldie, Cost-effectiveness of cervical cancer screening with human papillomavirus DNA testing and HPV16,18 vaccination, J Natl Cancer Inst 100 (2008), 308-320.

[9] H. Haapasalo, M. Kyläniemi, N. Paunul, V.L. Kinnula and Y. Soini, Expression of antioxidant enzymes in astrocytic brain tumors, Brain Pathol 13 (2003), 155-164.

[10] T. Hagemann, T. Bozanovic, S. Hooper, A. Ljubic, V.I. Slettenaar, J.L. Wilson, N. Singh, S.A. Gayther, J.H. Shepherd and P.O. Van Trappen, Molecular profiling of cervical cancer progression, Br J Cancer 96 (2007), 321-328.

[11] JC-m. Ho, S. Zheng, S.A. Comhair, C. Farver and S.C. Erzurum, Differential expression of manganese superoxide dismutase and catalase in lung cancer, Cancer Res 61 (2001), 85788585 .

[12] AK Holley, SK Dhar, Y Xu and D.K. St Clair, Manganese superoxide dismutase: beyond life and death, Amino Acids (2010), (ahead of print).

[13] T.S. Hwang, H.K. Choi and H.S. Han, Differential expression of manganese superoxide dismutase, copper/zinc superoxide dismutase, and catalase in gastric adenocarcinomas and normal gastric mucosa, Eur J Surg Oncol 33 (2007), 474-479.

[14] IARC - International Agency for Research on Cancer-World Health Organization. IARC Monographs on the evaluation of carcinogenic risks to humans: human papillomaviruses. 
Lyon: Human papillomavirus (2007). Vol. 90. Available from: <URL:http://www-dep.iarc.fr/>.

[15] R. Izutani, S. Asano, M. Imano, D. Kuroda, M. Kato and H. Ohyanagi, Expression of manganese superoxide dismutase in esophageal and gastric cancers, J Gastroenterol 33 (1998), 816-822.

[16] A.M. Janssen, C.B. Bosman, L. Kruidenier, G. Griffioen, C.B. Lamers, J.H. van Krieken, C.J. van de Velde and H.W. Verspaget, Superoxide dismutases in the human colorectal cancer sequence, J Cancer Res Clin Oncol 125 (1999), 327 335.

[17] A.M. Janssen, C.B. Bosman, W. van Duijn, M.M. Oostendorpvan de Ruit, F.J. Kubben, G. Griffioen, C.B. Lamers, J.H. van Krieken, C.J. van de Velde and H.W. Verspaget. Superoxide dismutases in gastric and esophageal cancer and the prognostic impact in gastric cancer, Clin Cancer Res 6 (2000), 31833192.

[18] F. Johnson and C Giulivi, Superoxide dismutases and their impact upon human health, Mol Aspects Med 26 (2005), 340352.

[19] V.L. Kinnula and J.D. Crapo, Superoxide dismutases in malignant cells and human tumors, Free Radic Biol Med 36 (2004), 718-744.

[20] V.L. Kinnula and J.D. Crapo, Superoxide dismutases in the lung and human lung diseases, Am J Respir Crit Care Med 167 (2003), 1600-1619.

[21] R. Liu, T.D. Oberley and L.W. Oberley, Transfection and expression of MnSOD cDNA decreases tumor malignancy of human oral squamous carcinoma SCC-25 cells, Hum Gene Ther 8 (1997), 585-595.

[22] X. Liu, A. Wang, L. Lo Muzio, A. Kolokythas, S. Sheng, C. Rubini, H. Ye, F. Shi, T. Yu, D.L. Crowe and X. Zhou, Deregulation of manganese superoxide dismutase (SOD2) expression and lymph node metastasis in tongue squamous cell carcinoma, BMC Cancer 10 (2010), 365.

[23] M. Malafa, J. Margenthaler, B. Webb, L. Neitzel and M. Christophersen, MnSOD expression is increased in metastatic gastric cancer, J Surg Res 88 (2000), 130-134.

[24] W.G. McCluggage, Endocervical glandular lesions: controversial aspects and ancillary techniques, J Clin Pathol 56 (2003), 164-173.

[25] L. Miao and D.K. St Clair, Regulation of superoxide dismutase genes: implications in disease, Free Radic Biol Med 47 (2009), 344-356.

[26] M.A. Moreira, A. Longatto Filho, A. Castelo, M.R. de Barros, A.P. Silva, P. Thomann, G. Mattosinho de Castro Ferraz Mda and G.B. Dores, How accurate is cytological diagnosis of cervical glandular lesions? Diagn Cytopathol 36 (2008), 270274.

[27] M. Ough, A. Lewis, Y. Zhang, M.M. Hinkhouse, J.M. Ritchie,
L.W. Oberley and J.J. Cullen, Inhibition of cell growth by overexpression of manganese superoxide dismutase (MnSOD) in human pancreatic carcinoma, Free Radic Res 38 (2004), 1223-1233.

[28] M. Schiffman, P.E. Castle, J. Jeronimo, A.C. Rodriguez and S. Wacholder, Human papillomavirus and cervical cancer, Lancet 370 (2007), 890-907.

[29] N.F. Schlecht, R.W. Platt, E. Duarte-Franco, M.C. Costa, J.P. Sobrinho, J.C. Prado, A. Ferenczy, T.E. Rohan, L.L. Villa and E.L. Franco, Human papillomavirus infection and time to progression and regression of cervical intraepithelial neoplasia, $J$ Natl Cancer Inst 95 (2003), 1336-1343.

[30] F.C. Schmitt, A Longatto-Filho, A Valent and P Vielh, Molecular techniques in cytopathology practice, J Clin Pathol 61 (2008), 258-267.

[31] Y. Soini, M. Vakkala, K. Kahlos, P. Paakko and V. Kinnula, MnSOD expression is less frequent in tumour cells of invasive breast carcinomas than in in situ carcinomas or non-neoplastic breast epithelial cells, J Pathol 195 (2001), 156-162.

[32] D. St Clair, Y. Zhao, L. Chaiswing and T. Oberley, Modulation of skin tumorigenesis by SOD, Biomed Pharmacother $\mathbf{5 9}$ (2005), 209-214.

[33] A.M. Svensk, Y. Soini, P. Pääkkö, P. Hiravikoski and V.L. Kinnula, Differential expression of superoxide dismutases in lung cancer, Am J Clin Pathol 122 (2004), 395-404.

[34] L. Termini, E. Boccardo, G.H. Esteves, R. Jr. Hirata, W.K. Martins, A.E. Colo, E.J. Neves, L.L. Villa and L.F. Reis, Characterization of global transcription profile of normal and HPV-immortalized keratinocytes and their response to TNF treatment, BMC Med Genomics 1 (2008), 1-29.

[35] L. Termini, P.C. Maciag, F.A. Soares, S. Nonogaki, S.M. Pereira, V.A. Alves, A. Longatto-Filho and L.L. Villa, Analysis of human kallikrein 7 expression as a potential biomarker in cervical neoplasia, Int J Cancer 127 (2010), 485-490.

[36] Y. Toh, S. Kuninaka, T. Oshiro, Y. Ikeda, H. Nakashima, H. Baba, S. Kohnoe, T. Okamura, M. Mori and K. Sugimachi, Overexpression of manganese superoxide dismutase mRNA may correlate with aggressiveness in gastric and colorectal adenocarcinomas, Int J Oncol 17 (2000), 107-112.

[37] T. Yan, L.W. Oberley, W. Zhong and D.K. St Clair. Manganesecontaining superoxide dismutase overexpression causes phenotypic reversion in SV40-transformed human lung fibroblasts, Cancer Res 56 (1996), 2864-2871.

[38] R.J. Zaino, Glandular lesions of the uterine cervix, Mod Pathol 13 (2000), 261-274.

[39] W. Zhong, L.W. Oberley, T.D. Oberley, T. Yan, F.E. Domann and D.K. St Clair, Inhibition of cell growth and sensitization to oxidative damage by overexpression of manganese superoxide dismutase in rat glioma cells, Cell Growth Differ 7 (1996), $1175-1186$. 


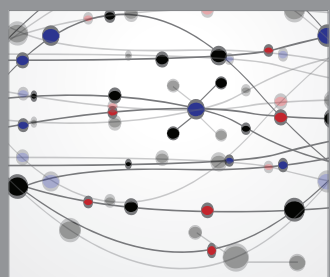

The Scientific World Journal
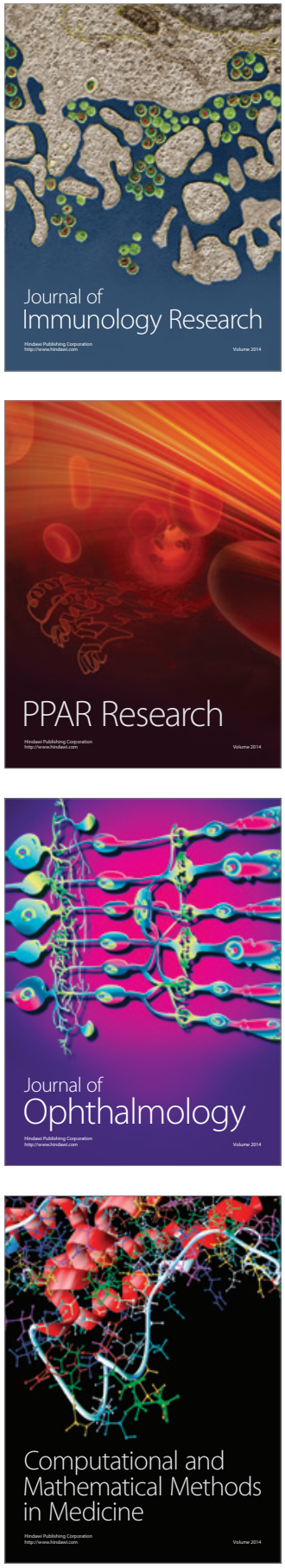

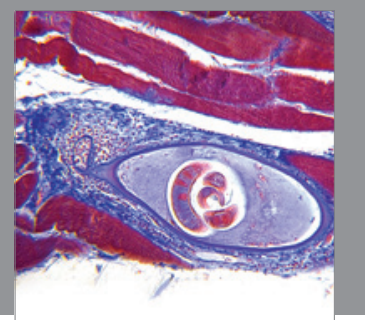

Gastroenterology

Research and Practice
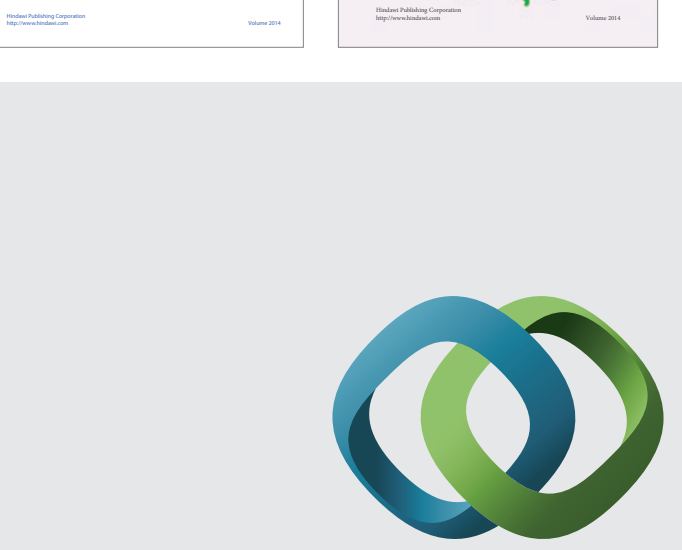

\section{Hindawi}

Submit your manuscripts at

http://www.hindawi.com
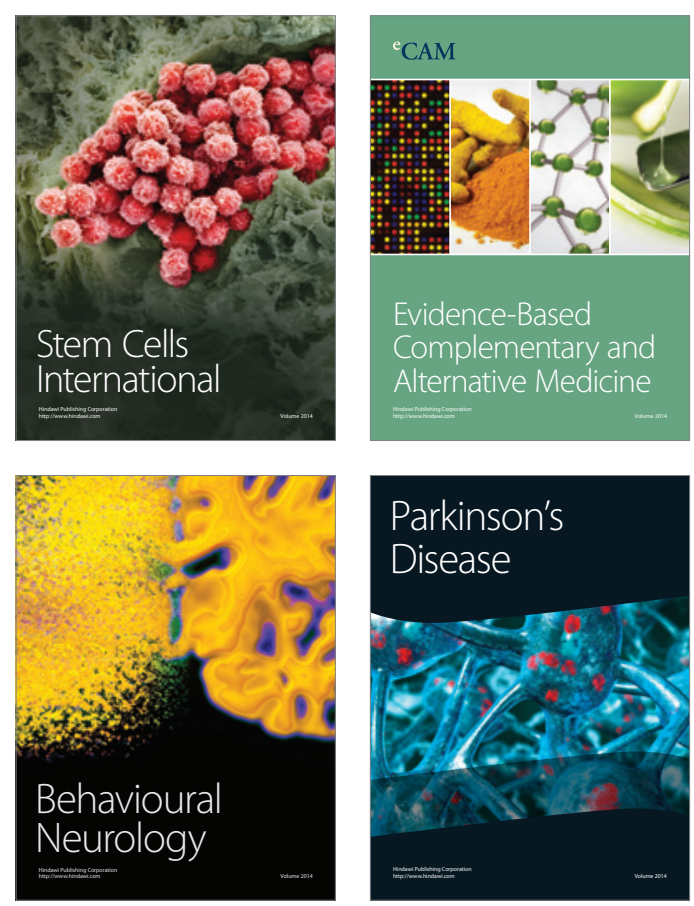

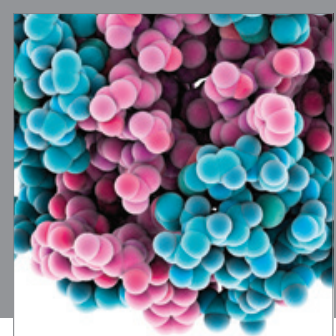

Journal of
Diabetes Research

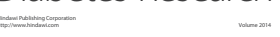

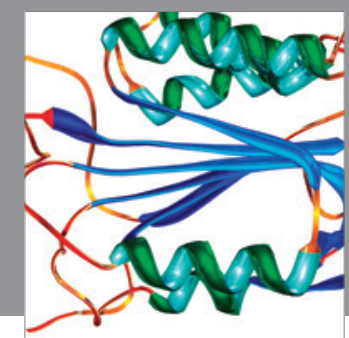

Disease Markers
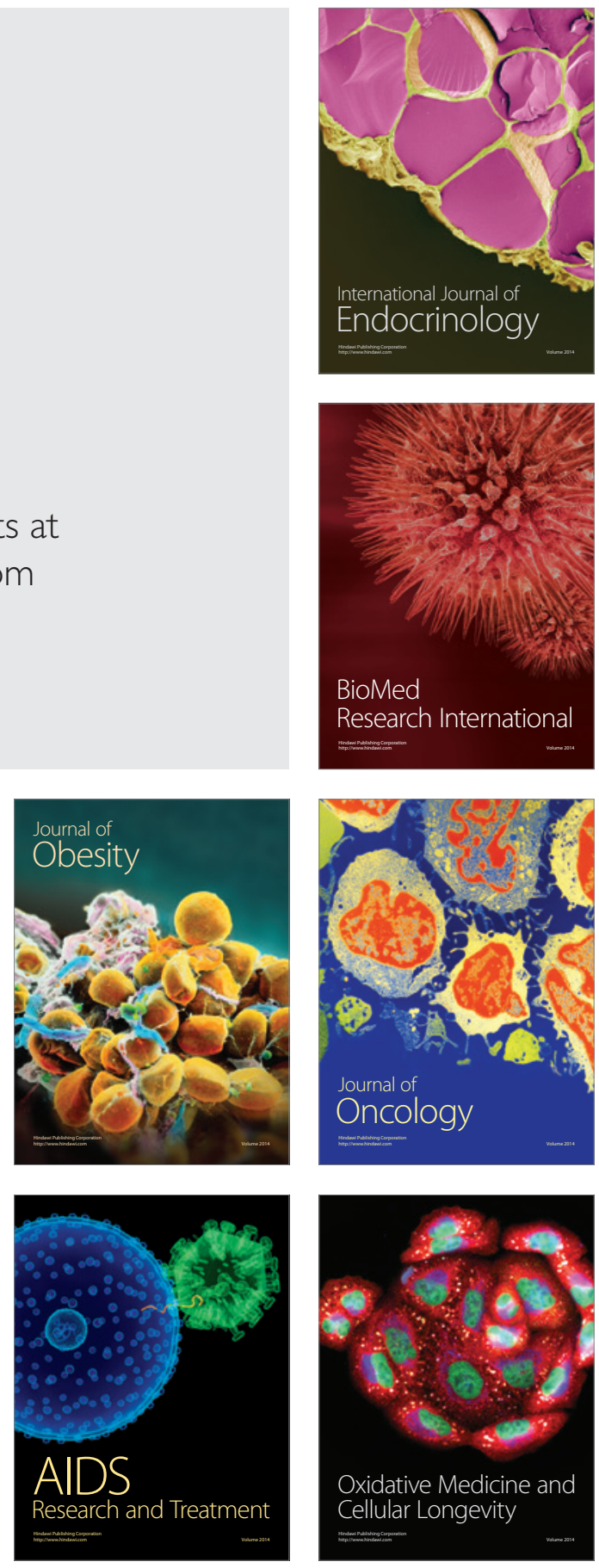